\title{
Criminologie
}

\section{De la pornographie légale à l'agression sexuelle}

Les scripts des activités des cyberdélinquants sexuels

From legal pornography to sexual assault

Sexual cyber-offender activity scripts

\section{De la pornografía legal a la agresión sexual}

\section{Los scripts de las actividades de los ciberdelincuentes sexuales}

\section{Francis Fortin, Sarah Paquette et Benoît Dupont}

Volume 50, numéro 1, printemps 2017

L'agression sexuelle commise sur des mineurs : les victimes, les auteurs

URI : https://id.erudit.org/iderudit/1039802ar

DOI : https://doi.org/10.7202/1039802ar

Aller au sommaire du numéro

Éditeur(s)

Les Presses de l’Université de Montréal

ISSN

0316-0041 (imprimé)

1492-1367 (numérique)

Découvrir la revue

Citer cet article

Fortin, F., Paquette, S. \& Dupont, B. (2017). De la pornographie légale à l'agression sexuelle : les scripts des activités des cyberdélinquants sexuels.

Criminologie, 50(1), 203-231. https://doi.org/10.7202/1039802ar

\section{Résumé de l'article}

La théorie des scripts permet une compréhension séquentielle de la criminalité sous différentes formes. Alors que traditionnellement les chercheurs se sont intéressés aux crimes contre la propriété puis aux crimes contre la personne, la présente étude vise l'analyse d'une nouvelle forme de criminalité, soit celle commise au moyen d'Internet. L'objectif de cet article est d'examiner, à partir d'une recension des écrits scientifiques, la manière dont la consommation de pornographie juvénile peut être comprise selon une perspective dynamique. Il y est avancé que le consommateur de pornographie juvénile motivé, en acquérant de nouvelles connaissances et techniques, traverse nombre d'étapes et d'obstacles le menant ultimement à l'agression sexuelle d'enfants. Il est toutefois important de noter que seule une petite partie des individus s'engageront dans les étapes subséquentes du script et que l'accent est mis sur le contexte et non sur les liens de causalité qui unissent les étapes puisque leur existence reste à démontrer. Plus précisément, les cheminements de la consommation de pornographie adulte à la pornographie juvénile, à la distribution de ce type de matériel, au leurre d'enfant et, enfin, au basculement dans le réel, soit l'agression d'enfant et la production de pornographie juvénile seront détaillés. Les limites et les implications de l'étude seront discutées.
Ce document est protégé par la loi sur le droit d'auteur. L'utilisation des services d’Érudit (y compris la reproduction) est assujettie à sa politique d'utilisation que vous pouvez consulter en ligne.

https://apropos.erudit.org/fr/usagers/politique-dutilisation/ 


\title{
De la pornographie légale à l'agression sexuelle
}

\section{Les scripts des activités des cyberdélinquants sexuels}

\author{
Francis Fortin ${ }^{1}$ \\ Professeur adjoint \\ École de criminologie, Université de Montréal \\ francis.fortin@umontreal.ca \\ Sarah Paquette \\ Doctorante \\ Département de psychologie, Université de Montréal \\ sarah.paquette@umontreal.ca \\ Benoît Dupont \\ Professeur titulaire \\ École de criminologie, Université de Montréal \\ Directeur scientifique du SERENE-RISC \\ Titulaire de la Chaire de recherche du Canada en sécurité, identité et technologie \\ benoit.dupont@umontreal.ca
}

RÉSUMÉ - La théorie des scripts permet une compréhension séquentielle de la criminalité sous différentes formes. Alors que traditionnellement les chercheurs se sont intéressés aux crimes contre la propriété puis aux crimes contre la personne, la présente étude vise l'analyse d'une nouvelle forme de criminalité, soit celle commise au moyen d'Internet. L'objectif de cet article est d'examiner, à partir d'une recension des écrits scientifiques, la manière dont la consommation de pornographie juvénile peut être comprise selon une perspective dynamique. Il y est avancé que le consommateur de pornographie juvénile motivé, en acquérant de nouvelles connaissances et techniques, traverse nombre d'étapes et d'obstacles le menant ultimement à l'agression sexuelle d'enfants. Il est toutefois important de noter que seule une petite partie des individus s'engageront dans les étapes subséquentes du script et que l'accent est mis sur le contexte et non sur les liens de causalité qui unissent les étapes puisque leur existence reste à démontrer. Plus précisément, les cheminements de la consommation de porno-

1. École de criminologie, Université de Montréal, C. P. 6128, succ. Centre-ville, Montréal (Québec), Canada, H3C 3J7.

Criminologie, vol. 50, $\mathrm{n}^{\circ} 1$ (2017) 
graphie adulte à la pornographie juvénile, à la distribution de ce type de matériel, au leurre d'enfant et, enfin, au basculement dans le réel, soit l'agression d'enfant et la production de pornographie juvénile seront détaillés. Les limites et les implications de l'étude seront discutées.

MOTS CLÉS • Cyberdélinquants sexuels, scripts, pornographie juvénile, agresseurs sexuels d'enfants.

\section{Introduction}

Les développements technologiques comme l'informatisation de presque toutes les sphères de l'activité humaine et l'omniprésence d'Internet offrent maintenant de nouvelles possibilités aux délinquants. La vaste majorité des études recourent aux typologies pour décrire la délinquance sur Internet, supposant que les types de consommateurs de pornographie juvénile restent statiques au fil du temps, comme si aucune migration n'était possible entre les types d'infracteurs (Krone, 2004; Lanning, 2010). Or, une telle analyse taxonomique pourrait s'avérer insuffisante pour bien comprendre les comportements des cyberdélinquants sexuels (Aslan, 2011). De plus, très peu d'études ont examiné les prédicteurs du passage à l'acte avec contact des consommateurs de pornographie juvénile (Lee, Li, Lamade, Schuler et Prentky, 2012; Long, Alison et MacManus, 2013). Conséquemment, on ne saurait encore dire quels mécanismes et éléments sont à considérer pour qu'un consommateur puisse passer d'apprenti à expert, ou même pour qu'il bascule dans l'agression sexuelle. La présente étude tente de définir certains scénarios délictuels en prenant comme matière première l'analyse des études sur la pornographie juvénile. Cette étude puisera son cadre analytique dans la théorie des scripts criminels (Cornish, 1994), en portant une attention particulière à la façon dont les évènements et les épisodes se déroulent pour les cyberdélinquants sexuels.

Un produit des sciences cognitives, les scripts sont définis comme des métatypes décrivant la manière dont les évènements surviennent normalement (Schank et Abelson, 1977). L'approche des scripts adaptée à l'acte criminel propose un moyen de générer, d'organiser et de systématiser les connaissances sur les aspects procéduraux et routiniers de la commission du crime (Cornish, 1994). Cette approche est utile pour l'analyse des processus de la commission du crime et aide à cerner l'ensemble des décisions et des actions des délinquants en découpant chaque étape de leurs crimes. Cornish (1994) évoque les différentes 
étapes criminelles: la sélection de la cible, la préparation, la commission de l'acte, l'évasion et l'après-crime. Ainsi, dans une situation particulière, les individus ont tendance à adopter une séquence d'essais et erreurs afin d'obtenir les résultats escomptés. Les scripts sont donc la description, étape par étape, de la procédure qui conduit les délinquants à réussir leurs crimes (Leclerc, Wortley et Smallbone, 2011).

On distingue quatre niveaux d'analyse des scripts criminels (Cornish, 1994). D’abord, le métascript comprend tous les crimes inclus dans une même classe, notamment l'agression sexuelle qui désigne une pluralité d'infractions. Le protoscript se situerait dans les sous-groupes de délinquants (p. ex. : agressions sexuelles d'enfants). Quant au script, il correspond à l'infraction qui est découpée en catégories pertinentes dans le crime à l'étude et qui s'avérera utile dans la prévention situationnelle, qu'elle soit liée à la victime, à la situation ou au modus operandi. Finalement, le niveau de la piste (track) constitue le niveau d'abstraction le plus bas et le plus pragmatique. L'objectif de développer différents niveaux de scripts criminels permet de fournir des descriptions plus détaillées et plus utiles des processus propres à la prévention du crime.

\section{L'application du cadre analytique des scripts criminels utilisé dans d'autres études}

Les criminologues ont largement employé les scripts criminels afin de mieux comprendre les crimes comportant une dimension technique importante. D’abord utilisés dans l'étude des crimes contre la propriété (Cornish, 1994; Morselli et Roy, 2008), les scripts ont ensuite été appliqués aux crimes contre la personne. Par exemple, des auteurs ont analysé les scripts des agresseurs sexuels (Beauregard, Proulx, Rossmo, Leclerc et Allaire, 2007; Deslauriers-Varin et Beauregard, 2010; Leclerc et al., 2011), des attaquants de type kamikaze (suicide bomber) (Clarke et Newman, 2006) et des auteurs d'actes de piraterie routière (carjacking) (Copes, Hochstetler et Cherbonneau, 2012). Les études montrent que les scripts sont facilement permutables selon les contingences de la situation. Il semble que certains criminels commettent leurs délits suivant une approche qui s'avérera, selon une analyse coût-avantage, la plus efficace. Ces travaux ne semblent toutefois pas prendre en compte le caractère dynamique des délinquants, qui passent d'une forme de script à une autre suivant la progression de leur expertise, mais aussi leur cheminement vers des formes plus graves de criminalité. 


\section{L'application du cadre des scripts dans les activités des cyberdélinquants sexuels}

L'utilisation des scripts criminels dans l'analyse des activités de consommation de pornographie juvénile nécessite quelques précisions et ajustements. D'abord, il paraît nécessaire d'adapter et de préciser un élément conceptuel des scripts tel qu'il est proposé par Schank et ses collaborateurs (1977). En effet, les lieux sont une composante déterminante dans le déroulement d'un script donné. Si les caractéristiques de l'endroit où se produit le crime a une influence fondamentale sur le script, il est essentiel de mentionner que la présente étude recense des actions se déroulant initialement dans un espace virtuel. Il importe donc de spécifier que les sites Web et les outils qui y sont associés peuvent être considérés comme des «lieux» dans la mesure où les individus décident de les visiter. Ainsi, les lieux virtuels supposent des propriétés relationnelles différentes de celles qui caractérisent les espaces physiques, notamment à l'égard de l'anonymat, de la capacité à se projeter simultanément dans plusieurs champs d'application différents, ou encore à recourir à des techniques de camouflage et de cryptage qui sont difficilement accessibles dans les lieux physiques (voir Castells, 2002).

Par ailleurs, cette étude présente une particularité au sens où le temps revêt une importance dans la compréhension de cette criminalité. En effet, il semblerait que chaque nouvelle image de pornographie juvénile constitue un crime. Puisque le temps consacré au téléchargement d'une image est très court, qu'il peut être en partie automatisé et qu'il fait partie d'un ensemble de comportements entourant les activités de collection, la présente analyse se positionnera à un niveau plus élevé. L'analyse des activités de consommation de pornographie juvénile adopte donc une vision temporelle beaucoup plus longitudinale par opposition à l'urgence et à la nature immédiate d'une situation, si on considère par exemple la réaction d'une victime, dans une fraction de seconde, lors d'un épisode de piraterie routière (Copes et al., 2012). La conception des cyberdélinquants place donc la dimension temporelle au cœur de l'analyse. Ainsi, les épisodes criminels sont distribués sur une échelle de gravité et selon une séquence temporelle plus longue. Cette conception met l'accent sur l'escalade qui caractérise l'enchaînement de divers épisodes et les conditions pour que cet enchaînement se réalise.

Le concept de métascript sera utilisé puisqu'il implique une grande catégorie de crimes, soit les infractions liées à la consommation de 
pornographie juvénile. La manière dont ces délits se ramifient d'un script vers l'autre afin de former un ensemble, c'est-à-dire les scripts des activités des cyberdélinquants sexuels, sera expliquée.

\section{Implications de l'étude}

La présente étude s'inscrit dans une volonté d'expliquer le passage à l'acte dynamique d'un nombre restreint de délinquants sexuels, soit ceux qui ont déplacé leur criminalité de la consommation de pornographie juvénile à l'agression sexuelle d'enfants. Ainsi, il est important de noter que les études scientifiques semblent indiquer que seul un faible pourcentage des consommateurs de pornographie juvénile ont également commis des agressions sexuelles (p. ex.: Seto, Hanson et Babchishin, 2011). Ces études laissent plutôt entendre que la majorité des auteurs de délits pédopornographiques possèdent des caractéristiques psychologiques et sociales qui les protègent d'un passage à l'acte avec contact (voir Finkelhor, 1984). C'est donc dans ce contexte que la présente étude tentera, à la lumière des données scientifiques disponibles, d'expliquer le caractère dynamique des activités d'un sous-groupe spécifique de cyberdélinquants sexuels.

\section{Méthodologie}

Afin d'établir une liste d'événements possibles dans les activités des cyberdélinquants sexuels, une recension de la littérature scientifique liée aux thèmes de la pornographie juvénile et des activités reliées à l'exploitation sexuelle sur Internet a été effectuée. Des requêtes auprès de trois moteurs de recherche ont été effectuées (Sociological Abstract, PsycNET et Social Sciences Abstract) avec les mots clés suivants: child luring, online solicitation, child pornography, child grooming, sexual grooming, pornographie juvénile, leurre informatique. Les études se concentraient sur les articles scientifiques (revus par les pairs) publiés entre 1990 et 2014. Ensuite, une analyse a été effectuée afin de relever les doublons et d'évaluer la pertinence des études (Sociological abstract-Proquest $(n=67)$, PsycNET $(n=201)$ et EBSCO (Social Sciences Abstracts) $(n=118)$. En effet, plusieurs études relataient des sujets connexes: «sexting», points de vue légaux, préférences sexuelles déviantes dans la population, impacts sur les individus travaillant auprès de cette clientèle, etc. Des livres cités par les études sélectionnées dans lesquels figuraient 
des résultats empiriques ont également été inclus dans l'échantillon. Au total, une cinquantaine d'articles et d'ouvrages ont été retenus. À la lumière de cette recension, les différents comportements relatés dans les études ont été divisés sous forme d'épisodes. Finalement, le contexte dans lequel les individus passaient d'un épisode à l'autre a été noté. Cette approche s'apparente à celle de Clarke et Newman (2006), lesquels ont fait l'exercice de recréer les scripts sans disposer de données directes pour étudier les kamikazes terroristes.

Pour réaliser cette étude, les outils, le fonctionnement de ces derniers ainsi que les modus operandi des cyberdélinquants ont été recensés et attribués à trois grandes familles d'activités sexuelles en ligne. Une revue de la littérature a permis d'établir que les cyberdélinquants sexuels se distinguent selon trois catégories: l'explorateur, soit l'individu qui accède à des contenus pédopornographiques et les visionne; le distributeur, soit celui qui partage ces contenus; et l'agresseur, soit celui qui commet des gestes d'agression sexuelle sur un enfant (Corriveau et Fortin, 2011; Fortin et Roy, 2006; Klain, Davies et Hicks, 2001 ; Krone, 2004; McLaughlin, 2000; Strano, Germani, Gotti et Errico, 2003). Dans une méta-analyse sur les caractéristiques des consommateurs de pornographie juvénile, Babchishin et ses collaborateurs (2011) montrent quatre cas d'utilisation de ce type de contenus: (a) l'accès par curiosité ou sous le coup d'une impulsion, sans intérêt sexuel particulier pour l'enfant; (b) l'accès pour satisfaire les fantasmes sexuels, sans toutefois commettre d'infractions sexuelles avec contact; (c) le désir de créer et de distribuer de la pornographie juvénile uniquement par appât du gain ${ }^{2}$; et, enfin, (d) l'utilisation d'Internet pour faciliter les infractions sexuelles avec contact.

\section{Scripts, pornographie juvénile et lien avec le passage à l'acte}

L'époque est révolue où les contenus Web étaient facilement et ouvertement disponibles et où les contrôles étaient minimaux. Ainsi, pour un consommateur de pornographie juvénile motivé, il existe une étape d'apprentissage, constituée d'essais et erreurs, semblable à celle que pourraient expérimenter d'autres types de criminels. La recension des

2. Dans le cadre de la présente étude, il a été décidé d'exclure le phénomène des réseaux commerciaux de distribution de pornographie juvénile puisque celui-ci est, à ce jour, encore trop peu étudié et suppose un objectif bien différent. 
différentes études a permis d'établir un schéma dynamique des scripts impliquant l'exploitation sexuelle des enfants sur Internet. Le modèle présenté à la Figure 1 se distingue par la présence de quatre épisodes. D'abord, à l'intérieur de chaque épisode, l'internaute intègre une série d'apprentissages concernant les lieux virtuels et les outils utiles. Le contexte du cyberespace permet de croire que l'apprentissage social (Sutherland, 1947) peut survenir différemment lorsqu'on est en ligne. Selon Ouimet (2006), Internet fourmille d'informations, parmi lesquelles on retrouve les traces indélébiles qu'y laissent les interactions entre individus. Or, l'application des notions d'apprentissage social à l'ère d'Internet implique que les criminels virtuels ne font pas leur apprentissage hors ligne auprès de pairs délinquants, mais bien dans les forums de discussion virtuels. Ainsi, en consultant le contenu de conversations archivées sur un forum de discussion asynchrone, l'internaute néophyte par rapport au milieu criminel aura accès à de nouvelles connaissances. L'anonymat que procure Internet favorise la confiance des internautes qui n'hésiteront pas à discuter en toute liberté (Corriveau, 2010; voir ces auteurs pour des exemples: Ghernaouti-Helie, 2007; Holt, Blevins et Burkert, 2010; Prichard, Watters et Spiranovic, 2011).

Ensuite, il a été établi que des barrières doivent être franchies afin d'atteindre l'épisode suivant. Lorsque l'apprentissage sera adéquat et que les conditions préalables seront remplies, un nouvel épisode prendra place, entraînant des changements dans les nouveaux apprentissages, dans les contenus et les actions posées par le délinquant. L'analyse et la description de ces points de basculement seront plus amplement détaillées dans les sections à venir. Ces barrières ont été inspirées de celles évoquées dans le modèle des conditions préalables à l'agression sexuelle (Finkelhor, 1984). Selon ce modèle, l'agression sexuelle s'explique par quatre facteurs distincts, nécessaires et séquentiels: une motivation à agresser, une propension à surmonter les inhibitions internes et les barrières externes ainsi que la résistance de la victime. Ces barrières constituent le dernier rempart avant l'agression sexuelle déjà alimentée par la préférence sexuelle déviante existante.

\section{Présentation du métascript associé aux activités de consommation de pornographie juvénile sur Internet}

L'épisode 1 s'ouvre sur une étape initiale d'exploration des contenus pornographiques sur Internet. La première barrière à franchir consistera donc à étendre l'exploration vers la pornographie juvénile. Puis, le sujet 
F I G U R E 1

Analyse du métascript du consommateur de pornographie juvénile

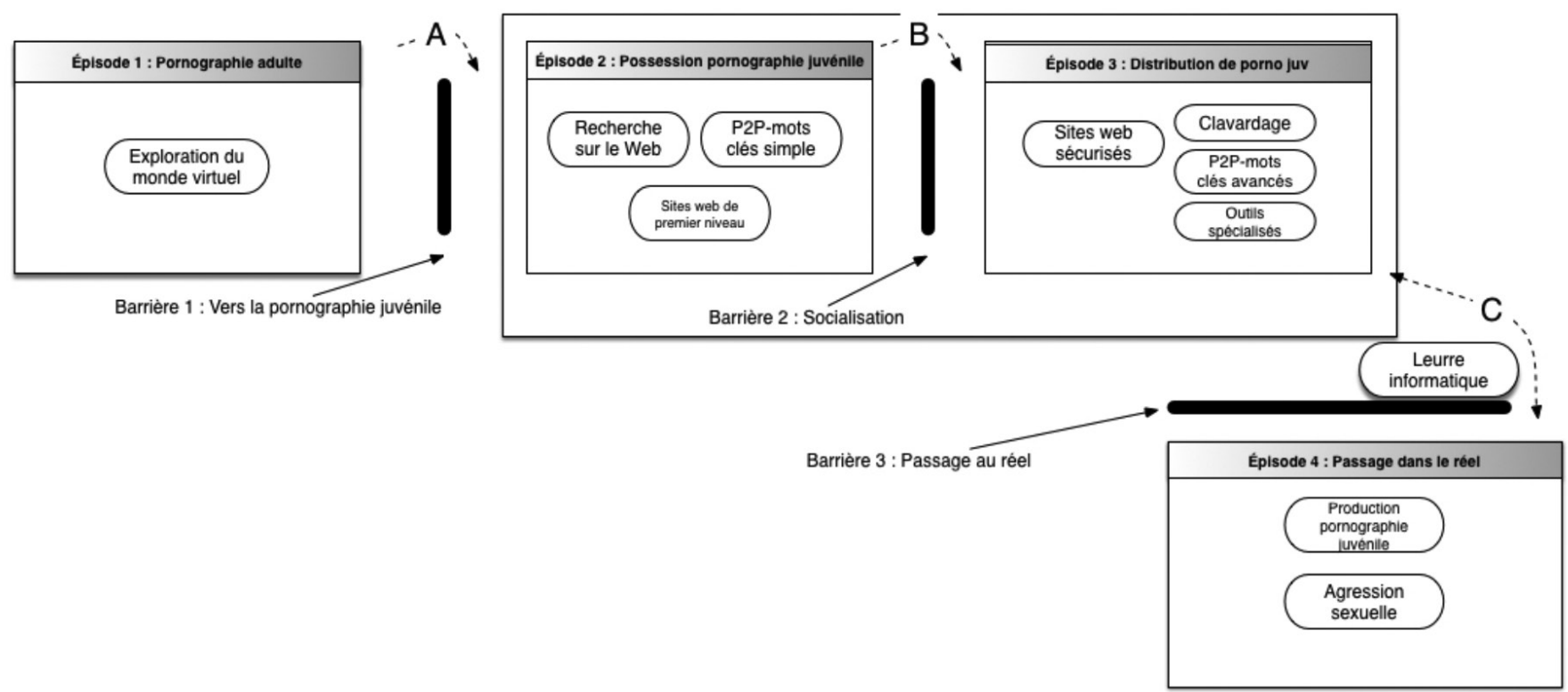


explorera les premières possibilités d'Internet en utilisant des moteurs de recherche et des lieux virtuels aisément accessibles pour accéder à des contenus. Ainsi, pour obtenir des contenus inédits ou plus intéressants, il doit nécessairement franchir la barrière de la socialisation virtuelle. C'est grâce à l'observation des comportements des autres consommateurs, en discutant virtuellement et aussi en partageant des contenus que le cyberdélinquant pourra obtenir de nouveaux contenus plus exclusifs que ceux qui sont facilement accessibles et disponibles. Puis, lorsque le matériel virtuel ne suffira plus, il devra évaluer la possibilité de franchir la barrière du passage à l'acte dans le réel. L'épisode 4 démarrera ensuite lorsque la sollicitation sexuelle des enfants, ou encore l'agression sexuelle, aura lieu.

\section{Épisode 1: de la pornographie légale à la pornographie juvénile $(A)$}

La démocratisation d'Internet a favorisé l'accès à des contenus pornographiques de tous genres. Parmi les cas d'individus qui consultent régulièrement de la pornographie, $7 \%$ relèveraient d'un diagnostic de sexualité compulsive (Cooper, Delmonico et Burg, 2000; Philaretou, Mahfouz et Allen, 2005), s'accompagnant d'effets et de résultats négatifs, notamment la dépression, l'anxiété et les difficultés relationnelles et intimes (Philaretou et al., 2005; Twohig, Crosby et Cox, 2009). Cette problématique a également été observée chez des consommateurs de pornographie juvénile (Griffin-Shelley, 2014). Par ailleurs, en plus de la dépendance aux contenus pornographiques, il a été démontré que certains consommateurs de pornographie juvénile présentaient une dépendance au média lui-même, Internet (Lee et al., 2012).

Dans une perspective similaire à celle présentée dans cette étude, Taylor et Quayle (2003) ont plutôt tenté d'appliquer un modèle développemental en tenant compte de la progression des individus vers la consommation de pornographie juvénile. Les auteurs ont tenté d'établir un modus operandi typique de ce type de cyberdélinquants, passant par un certain nombre d'étapes d'engagement en ce qui concerne Internet. Les auteurs ont établi que le point de départ du cheminement serait la pornographie légale. En entrevue, des personnes arrêtées relativement à des délits de pornographie juvénile ont affirmé que le premier contact avec Internet a souvent débuté par l'accès à des sites pornographiques pour adultes et leur exploration s'est poursuivie avec la recherche de 
pornographie juvénile (Roy, 2004; Taylor, 2001). Le modèle de Taylor et Quayle (2003) soutient l'idée selon laquelle les comportements délictueux d'un certain nombre de cyberdélinquants sexuels s'intégreraient dans un processus temporel dynamique.

\section{Obstacle A: vers la pornographie juvénile}

$\mathrm{Si}$, pour certaines personnes, la pornographie adulte constitue une porte d'entrée vers les contenus de pornographie juvénile, le temps passé à mieux connaître ce milieu virtuel aboutira inévitablement à d'autres types d'offres. Dans une étude sur l'utilisation des moteurs de recherche afin de trouver des contenus pédopornographiques, Corriveau et Fortin (2011) ont montré qu'il était difficile de naviguer sur des sites de redirection, montrant que cette navigation nécessite des apprentissages. De plus, au cours de cette exploration, l'offre vers des images de sujets plus jeunes arrivera inévitablement. L'individu verra que l'offre est bien présente et, en apparence, elle ne paraît pas plus difficile à obtenir que la pornographie légale. En effet, certains sites utilisent l'attrait des contenus "barely legal», ou encore mélangent des images d'adolescents des deux sexes avec un contenu principalement adulte. Cette tendance à proposer des images de jeunes a été étudiée en analysant des pochettes de DVD offertes sur le marché (Jensen, 2010). Plus de $20 \%$ des pochettes contenaient des termes ou des images évoquant la jeunesse.

\section{Épisode 2: la possession de pornographie juvénile}

Des chercheurs ont étudié la quête d'un individu pour constituer une collection dans un contexte tout à fait légal (Belk, 1994; Belk, Wallendorf, Sherry et Holbrook 1991; Formanek, 1991). L'aspect central de la collection réside dans la constante acquisition de nouveaux objets prisés (Muensterberger, 1994). Collectionner des objets aurait pour de nombreux amateurs un effet de dépendance dans la mesure où l'ajout de nouveaux articles à la collection procurerait ce que les études sur la dépendance appellent une «dose» ou un «fix» (Belk, 1994).

Le fait de collectionner du matériel de pornographie juvénile incite certains individus à vouloir augmenter sans cesse le volume de leur collection (Lanning et Burgess, 1984) et à acquérir du matériel dont le contenu devient de plus en plus extrême. Le processus est très minutieux, ne laissant rien au hasard, les acquisitions étant classées logique- 
ment selon une variété de critères (par groupes d'âge, sexe, types d'actes, etc.) (McLaughlin, 2000). Le plaisir repose sur les activités entourant la collection (Carr, 2004) ainsi que sur la difficulté et l'effort associés à la recherche d'une image manquante d'une série que le collectionneur possède, même si le matériel n'est pas nécessairement attirant ou excitant pour ce dernier (Quayle et Taylor, 2002).

Le script de l'apprenti collectionneur se distingue par des démarches d'exploration du consommateur solitaire (c.-à-d. dont la visée n'est pas l'acquisition systématique de matériel de pornographie juvénile). Dans une étude reproduisant les actions d'un néophyte à la recherche de matériel pédopornographique grâce aux moteurs de recherche courants tels que Google, des chercheurs ont tenté de trouver des contenus de pornographie juvénile à partir de mots clés simples (Corriveau et Fortin, 2011). Les résultats se sont avérés très peu fructueux, $5 \%$ des sites ciblés comportant des images d'adolescents. Ce résultat montre que l'acquisition de matériel de pornographie juvénile nécessite un certain effort pour y parvenir, contredisant ainsi la thèse de l'accès par inadvertance. Ils concluent aussi que la distribution de ce matériel ne semble pas s'effectuer principalement par l'entremise des moteurs de recherche traditionnels. C'est aussi ce qu'a observé Steel (2009) qui a découvert qu'entre 2004 et 2008 les requêtes sur les moteurs de recherche pour des termes associés à la pornographie juvénile ont diminué d'environ $60 \%$. Selon Temporini (2012), l'explication la plus probable serait que la médiatisation des opérations policières aurait donné lieu à un changement de lieu virtuel pour les consommateurs de pornographie juvénile. Il y aurait donc eu un déplacement vers les outils de recherche qui renforcent la perception d'anonymat de l'utilisateur, tels les réseaux poste-à-poste (P2P). De nombreux fichiers de pornographie juvénile sont ainsi échangés par l'entremise des technologies P2P (Carr, 2001; Wortley et Smallbone, 2006) et, en examinant les mots clés utilisés sur les réseaux $\mathrm{P} 2 \mathrm{P}$, une étude révèle que certains termes reflètent la présence indubitable du milieu de la pornographie juvénile sur ces plateformes d'échanges (Steel, 2009). Pour plusieurs usagers, l'apprentissage de mots clés pertinents peut se faire en analysant les résultats de recherche. À l'instar de la recherche de contenus légitimes, certains auteurs ont souligné l'interaction dynamique et itérative entre le besoin, l'information, le chercheur et l'environnement de l'information (voir Knight et Spink, 2008). L'exemple du mot clé PTHC ("preteen hard core») amène Steel (2009) à conclure que «cela indique un niveau de 
sophistication chez ceux qui cherchent de la pédopornographie sur les réseaux poste-à-poste en utilisant la terminologie propre à la sousculture» (p. 563).

Obstacle B : la socialisation virtuelle pour obtenir de meilleurs contenus

Le consommateur (solitaire ou collectionneur) de pornographie juvénile ne peut rester passif dans ses tâches de recherche. D'abord parce que le Web ne constitue pas une source intéressante pour un individu désirant nouer des contacts interpersonnels (Corriveau et Fortin, 2011). Ensuite, le collectionneur ne pourra continuer son apprentissage seul: il devra interagir avec les autres amateurs, apprendre les mots clés du milieu, connaitre les lieux virtuels ou les outils requis pour trouver plus efficacement des contenus d'intérêt (Corriveau et Fortin, 2011). Ce besoin de posséder de nouvelles images s'observe aussi chez les autres collectionneurs, incitant les amateurs à participer activement à divers réseaux d'échanges (Fortin, 2013). Les contenus désormais archivés peuvent être également utilisés comme référence ou moyen de négocier pour obtenir du nouveau matériel (Quayle et Taylor, 2002). Cette visibilité accrue dans les communautés virtuelles augmente par ailleurs les risques d'être repéré par les services policiers qui surveillent ces lieux d'échanges (Krone, 2004). Or, la distribution est nécessaire pour que le cyberdélinquant puisse obtenir du nouveau matériel ou, encore mieux, du matériel inédit. Ce dernier devra donc franchir la deuxième barrière, soit celle de la socialisation avec les autres consommateurs de pornographie juvénile.

\section{Épisode 3: de «simple» collectionneur à collectionneur et distributeur}

Les activités de recherche de pornographie juvénile sur Internet en solitaire amènent certains individus à s'impliquer davantage dans la communauté de pairs qui partagent les mêmes champs d'intérêt. L'épisode 3 débute lorsque la socialisation virtuelle s'enclenche. L'investissement en temps dans les activités en ligne se fait alors au détriment d'activités extérieures. Ces concepts sont illustrés dans l'étude de Taylor, Quayle et Holland (2001) dont la schématisation conceptuelle est présentée dans la Figure 2. 
FIGURE 2

Étapes opératoires selon Taylor et al. (2001)

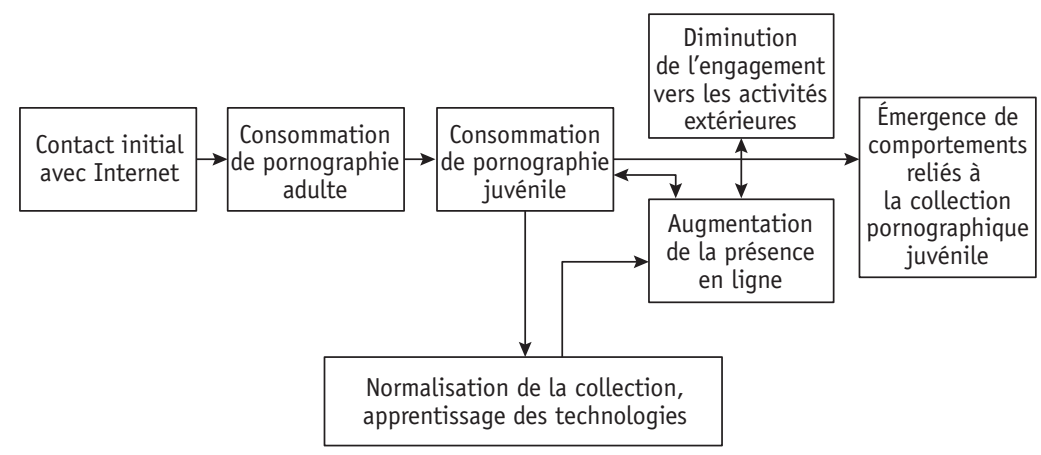

Comme discuté précédemment, la consommation de pornographie adulte précède pour certains celle de la pornographie juvénile. Par la suite, un cycle débute là où l'augmentation de la présence en ligne est inversement proportionnelle à l'engagement dans des activités extérieures. Une plus grande présence en ligne multiplie aussi les contacts avec d'autres individus, normalise les activités de collection et contribue à l'apprentissage des moyens techniques pour favoriser l'ajout de pièces à la collection. La question de l'apprentissage individuel et social (voir Akers, 2011; Sutherland, 1974) est au cœur des étapes développementales du collectionneur. Après la création de grandes collections et grâce aux connaissances et aux habiletés acquises, des pratiques telles que le tri, le classement et la catégorisation du matériel prennent une place de plus en plus importante dans la vie du collectionneur. Selon Taylor et Quayle (2003), la délinquance dans ce contexte est un processus dynamique plutôt que statique au sens où les individus se positionnent différemment le long d'un continuum de développement par rapport à la pornographie juvénile. Ce positionnement est fonction de la satiété de l'excitation sexuelle, des processus d'engagement dans la collection et dans les communautés virtuelles ainsi que dans l'exploration de différentes identités en ligne.

\section{Le script du distributeur}

Le script du distributeur nécessite un apprentissage et la maitrise de la diffusion de ces contenus illicites à une ou plusieurs personnes par l'entremise de différents moyens technologiques. Les cyberdélinquants distribueront leurs images dans différents lieux (services), notamment 
des communautés virtuelles (privées ou publiques), des forums de discussion, ou encore par l'entremise de la messagerie instantanée.

Les salons de clavardage permettent aux utilisateurs d'entrer directement en contact les uns avec les autres pour effectuer leurs échanges de contenus pédopornographiques (Forde et Patterson, 1998; Fortin et Lapointe, 2002). Les comportements des autres internautes permettent d'ailleurs aux cyberdélinquants de justifier leurs propres activités illicites sur Internet (Houtepen, Sijtsema et Bogaerts, 2014). En 1997, les chercheurs du projet COPINE ont étudié plusieurs de ces salons de clavardage (Taylor et Quayle, 2003). Ils ont constaté que 518 amateurs de pornographie juvénile y échangeaient des images pédopornographiques et s'adonnaient à des jeux de rôle mettant en scène des adultes jouant le rôle d'un enfant et d'un adulte (Carr, 2001; Wortley et Smallbone, 2006). Des chercheurs ont constaté que le clavardage IRC est le moyen d'échange le plus fréquemment utilisé par une majorité (plus des deux tiers) des personnes arrêtées pour possession et distribution de pornographie juvénile (Carr, 2004; Roy, 2004).

Les groupes de nouvelles sont connus pour être des lieux privilégiés d'échange de pornographie juvénile (Carr, 2001; Fortin, 2013 ; Sellier, 2003 ; Taylor et al., 2001). Dans une étude sur les échanges dans les communautés de cyberdélinquants sexuels, Corriveau (2010) affirme que les membres du groupe accordent un statut supérieur à ceux qui distribuent des contenus. Il observe aussi la pression constante que des membres exercent afin de garder les autres utilisateurs actifs. Le prestige dans le groupe serait ainsi attribué à ceux qui distribuent du matériel, même ancien. Toutefois, les producteurs et les distributeurs de nouveau matériel sont explicitement placés au sommet de la hiérarchie informelle. La sociabilité remplit donc un rôle de légitimation des comportements et vient renforcer le choix de ceux qui ont effectué la transition vers ce nouvel épisode. De plus, une analyse du matériel consommé a révélé que ceux qui avaient des interactions avec d'autres utilisateurs possédaient des images plus problématiques (c.-à-d. des images présentant des enfants plus jeunes dans des situations plus graves) que les consommateurs solitaires (Fortin, 2014).

L'interaction avec les autres utilisateurs entraine la nécessité de recourir aux techniques de dissimulation des activités. Les études analysant les dynamiques dans les forums de discussion sur Internet ont soulevé l'aspect pédagogique des interactions (Corriveau, 2010; Corriveau et Fortin, 2011; Fortin, 2013). Dans la typologie de Krone 
(2004), le collectionneur «sécuritaire» se caractérise par l'utilisation du cryptage et son implication dans des groupes qui cachent l'identité des utilisateurs et dont les membres sont tenus de fournir des images de pornographie juvénile pour pouvoir y adhérer.

\section{Obstacle C: le passage au réel}

La prochaine barrière à franchir sera celle du passage au réel. Bien que seulement une faible portion des cyberdélinquants sexuels franchisse cette barrière (voir Seto et al., 2011), il existe des scripts observables qui expliquent comment ce processus s'opère. Ces scénarios se distinguent tous par une volonté d'interagir non plus avec d'autres collectionneurs, mais bien d'entrer directement en contact avec l'objet même de la collection, soit l'enfant.

\section{Épisode 4: de consommateur/distributeur à agresseur sexuel}

La troisième barrière à franchir pour l'amateur de pornographie juvénile est celle du passage à l'acte dans la réalité. La Figure 3 présente les différents liens qui peuvent unir l'action de consommer de la pornographie juvénile et celle de passer à l'acte. De l'analyse de la littérature scientifique effectuée dans le cadre de cette étude, quatre scripts distincts émergent et permettent d'expliquer les comportements des délinquants qui s'engagent dans des activités sexuelles hors ligne.

La pornographie juvénile comme outil facilitant la commission de l'agression (C1)

La collection permet d'abord aux délinquants d'obtenir une gratification sexuelle par l'entremise des fantaisies sexuelles que leur procure ce type de matériel illicite. Certains chercheurs ont étudié les fonctions instrumentales des collections de pornographie juvénile. D'une part, elles seraient un moyen de séduire et de désinhiber les enfants (Fillieule et Montiel, 1997; Lanning, 2010). La présentation de matériel pornographique aurait une valeur pédagogique ou démonstrative en fonction des actes sexuels infligés à l'enfant. Dans une étude, 23,1\% des délinquants utilisaient de la pornographie adulte et $21,6 \%$ recouraient à la pornographie juvénile (17,5\% utilisaient les deux types) (Bennett et Gates, 1991). Ainsi, le fait de présenter à l'enfant des films l'amènerait à vaincre ses inhibitions et sa résistance par rapport aux contacts sexuels avec 
Schématisation de la relation entre pornographie juvénile et passage à l'acte
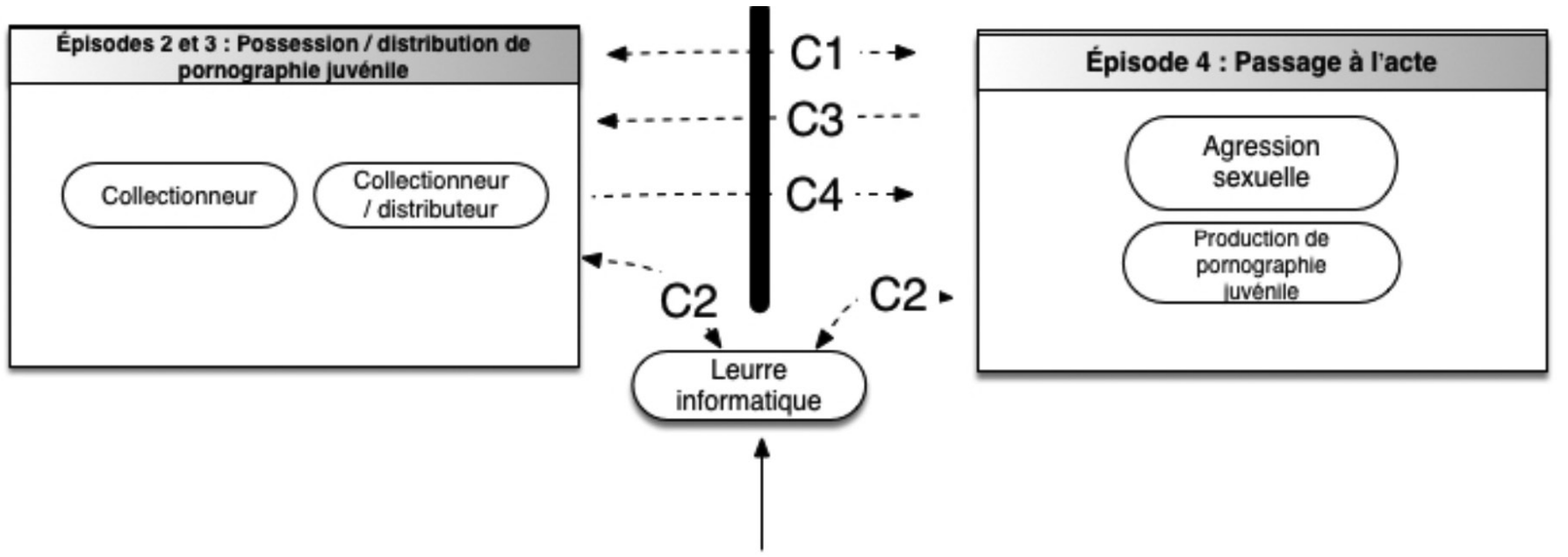

Barrière 3 : Passage au réel 
d'autres enfants, mais aussi avec des adultes (Fillieule et Montiel, 1997; Rettinger, 2000). Cette démarche contribuerait à normaliser ces comportements aux yeux des victimes (O'Brien, 1983; Rettinger, 2000). De plus, et contrairement aux autres formes d'actes criminels contre la personne, la plupart des délinquants emploient des techniques visant à persuader l'enfant de participer de son plein gré à l'infraction et espèrent que celui-ci en retire du plaisir (Proulx et Ouimet, 1995).

D'autre part, la consommation de pornographie juvénile peut être utilisée comme moyen de chantage. Pour une première infraction, par exemple, l'agresseur peut prendre des photos de l'enfant à différentes étapes de l'acte. Ces photos peuvent ensuite engager l'enfant plus avant dans le cercle vicieux de l'agression et le forcer à garder le silence sur l'agression subie (O’Brien, 1983 ; Skoog et Murray, 1998; Tate, 1990). Ayant la preuve numérisée de leurs actes, les agresseurs peuvent aisément menacer les enfants de montrer les photos qu'ils ont prises. Ils obtiendraient ainsi une coopération dans les tentatives subséquentes (Skoog et Murray, 1998). Les agresseurs ont donc intérêt à appliquer les règles du secret auprès de l'enfant avec lequel ils désirent entrer en contact, en plus d'isoler l'enfant des personnes qui pourraient le protéger. Comme le relève Ouellet (2008), les outils de messagerie instantanée, qui impliquent une communication plus intime, représentent un moyen de garder la relation secrète. On observe que ce passage vers l'intimité accroît la possibilité de discuter de sujets plus sexuels (Quayle et Taylor, 2001).

\section{Le script de l'auteur de leurre (C2)}

Certains individus vont utiliser les outils interactifs disponibles sur Internet afin de solliciter des victimes potentielles. Ce sont les mêmes services de clavardage utilisés pour discuter avec les complices qui seront utilisés pour faciliter la recherche de personnes susceptibles de répondre à des avances sexuelles (End child prostitution, child pornography and trafficking of children for sexual purposes [ECPAT], 2002; Fortin et Lanthier, 2013; Fortin et Lapointe, 2002). Dans le cadre de la présente étude, la sollicitation sexuelle d'enfant au moyen d'Internet (leurre) a été positionnée à mi-chemin entre la pornographie juvénile et l'agression sexuelle, puisque la documentation scientifique laisse supposer que certains individus entretiendront des relations avec des adolescents dans un contexte fait uniquement de fantaisies sexuelles (Briggs, Simon et Simonsen, 2011; DeHart et al., 2016). Il y aurait donc 
nombre d'individus qui continueraient à entretenir des fantasmes en interagissant directement avec l'objet de leur désir, tout en ne passant pas à l'acte.

Des chercheurs ont tenté de comprendre quels étaient les comportements des individus dans les salons de clavardage. Briggs, Simon et Simonsen (2011) ont analysé les données cliniques d'entrevues de 51 cyberdélinquants sexuels ainsi que les transcriptions de clavardage de ces personnes afin de définir des comportements typiques. L'analyse leur a permis d'observer deux groupes distincts, soit les individus motivés par les contacts sexuels dans la réalité («contact-driven») et les autres, motivés par le fantasme ( fantasy-driven»). Le premier groupe souhaiterait avoir des contacts sexuels hors ligne avec un adolescent. Internet leur permettrait d'obtenir des coordonnées avec une plus grande efficacité que la recherche dans les espaces publics. Le second groupe serait plus enclin à s'engager avec un adolescent dans une relation de cybersexe, sans intention de le rencontrer hors ligne. Selon les auteurs, il existerait un tronc commun aux deux types à l'étape de recherche des adolescents avec lesquels les délinquants souhaitent communiquer (clavardage, localisation de la victime, établissement du contact et introduction de contenu sexuel dans la conversation en envoyant entre autres une image du sujet nu à la victime). Pour le type "fantasy-driven», la masturbation, l'enseignement de la masturbation et les activités sexuelles en ligne sont beaucoup plus courantes (respectivement $76,2 \%, 66,7 \%, 81,0 \%$ de l'échantillon) que chez le type «contact-driven» qui, par définition, est probablement plus patient, ou peut-être moins intéressé par des activités virtuelles puisqu'il vise plutôt le transfert de la relation vers le réel dès que possible.

Il est possible que l'individu motivé par le fantasme puisse ne se trouver qu'à l'ultime étape avant de manifester un intérêt plus marqué pour les contacts physiques. Les échanges virtuels pourraient alors constituer un dernier rempart avant la rencontre dans le réel. Il ne faut pas exclure que des séances de cybersexe ou de masturbation en ligne puissent être un prélude à une agression sexuelle physique, transformant ainsi la personne motivée par le fantasme en un individu motivé par le contact sexuel en attente ou tout simplement temporairement assouvi. Par ailleurs, pour le cyberdélinquant motivé par le fantasme, les séances de clavardage pourraient s'avérer une prolongation de son attrait pour les images fixes d'adolescents. En obtenant une interaction avec ces jeunes lors de séances de webcam ou de cybersexe et en obtenant leurs 
photos, il est possible qu'il poursuive dans la fantaisie. Il demeure toutefois qu'échanger dans des salons de clavardage ouvre la porte à des opportunités criminelles en offrant la possibilité de créer des relations et des rencontres en personne (Wolak, Mitchell et Finkelhor, 2003).

On retrouve des images de pornographie juvénile chez plusieurs délinquants sexuels qui utilisent les salons de clavardage (Mitchell, Wolak et Finkelhor, 2005). Cela peut refléter un intérêt déjà présent, mais peut aussi résulter de demandes adressées à l'adolescent pour qu'il fournisse une photo de lui. Cela peut donc constituer un moyen additionnel d'obtenir des images de pornographie juvénile.

\section{Le script de l'agresseur producteur (C3)}

Le fait de produire de la pornographie juvénile représente un intérêt pour certains individus. Les études sur la production de ces contenus indiquent que le matériel de pornographie juvénile est produit de façon artisanale. Ces photos sont échangées ou réservées à l'usage personnel du producteur (Howitt, 1995 ; Rettinger, 2000). Dans une étude recensant les mécanismes de conservation de la preuve de leurs agressions, Warren, Dietz et Hazelwood (2013) concluent que les méthodes diffèrent chez les délinquants, mais qu'elles permettent notamment d'entretenir des rêveries en maintenant des souvenirs évocateurs ${ }^{3}$.

Bien que l'idée de pouvoir revivre l'agression et la relation avec l'enfant soit une motivation importante, il peut également arriver que le fait de pouvoir revendiquer les agressions devienne une motivation secondaire, notamment afin d'améliorer son statut au sein du groupe de pairs. Un cas semblable est évoqué dans l'étude de Corriveau et Fortin (2011), dans laquelle on rapporte que des internautes sceptiques exigeaient des preuves qu'il s'agissait bien de contenu exclusif. Dans un autre cas, un agresseur a pris une photo d'une victime tenant une feuille sur laquelle un message personnalisé avait été rédigé, suivant le modèle adopté par certains preneurs d'otages pour attester de la véracité de leurs exigences (Fortin et Lapointe, 2002). La photo fut envoyée aux internautes en guise de preuve.

3. Parmi les modalités de conservation, on trouve: la collecte de renseignements, la prise de trophées, la production de vidéos, la photographie, le récit d'histoires et la journalisation. 
Par ailleurs, alors que l'agression permettrait d'enrichir la collection, c'est également le besoin de collectionner qui inciterait à recommencer les agressions pour obtenir plus d'images (Lanning, 2010). Dans le cas du Wonderland Club, un réseau de cyberdélinquants, les adhésions étaient clairement réglementées: pour y être admis, les nouveaux membres passaient un examen rigoureux et devaient posséder une collection d'au moins 10000 images, différentes de celles déjà détenues par les anciens membres (ECPAT, 2007). Pour moins de $100 \$$ US par mois, les membres du réseau avaient accès aux fichiers pédopornographiques. La volonté de se faire reconnaître dans le milieu pouvait aussi être une motivation à produire du contenu. L'agression permettrait donc l'enrichissement de la collection, et le besoin de collectionner inciterait à recommencer les agressions pour obtenir de nouvelles images (Lanning, 2010). Ainsi, soit la collection reste la motivation principale de l'amateur, soit c'est l'envie d'agresser qui prédomine et qui incite à créer de nouvelles images par l'entremise de ces agressions sexuelles.

\section{Le script ponctuel de la personne excitée (C4)}

Le cas du meurtre d'Holly Jones au Canada constitue un exemple où, ponctuellement, un individu est passé à l'acte après avoir regardé de la pornographie juvénile. Dans ce cas extrême et rare, l'évènement s'est soldé par une agression sexuelle et un meurtre. Middleton, Beech et Mandeville-Norden (2009) estiment que le visionnement de pornographie peut être une source de stimulation des fantaisies sexuelles qui, dans certaines circonstances, tend à normaliser les comportements sexuels déviants et, potentiellement, conduit à l'agression. La dynamique du passage à l'acte peut varier d'un individu à un autre, voire chez un même individu en fonction des circonstances et de son environnement du moment. Selon les études, plus du tiers des agresseurs sexuels ont admis avoir regardé des magazines ou des films pornographiques avant au moins une de leurs infractions (Marshall, 1988). Proulx et ses collaborateurs (Proulx, Perreault et Ouimet, 1999) ont aussi analysé le déroulement des quelques heures ayant précédé le crime commis par 44 hommes incarcérés pour une agression extrafamiliale sur un enfant. Les résultats montrent que $25 \%$ de ceux-ci avaient fait usage de matériel pornographique juvénile. Dans ce sous-groupe, l'infraction était planifiée et des fantasmes sexuels déviants étaient présents. Ainsi, pour certaines personnes, la consommation de porno- 
graphie juvénile avant le crime ferait monter le désir et favoriserait le passage à l'acte.

\section{Conclusion}

La présente étude visait à expliquer les activités en ligne liées à la consommation de pornographie juvénile par l'application du cadre analytique des scripts criminels. La pertinence de l'emploi de ce cadre a été établie, notamment dans l'analyse des crimes contre la personne. À cet effet, il permet de modéliser plusieurs scénarios et cheminements possibles et de cerner les points de basculement d'un type de crime à un autre, plus grave.

Le premier épisode commence par la consommation de pornographie légale. L'intérêt pour ce contenu se transforme lorsque l'exploration des plateformes de distribution permet à l'internaute de découvrir qu'il est aussi possible d'accéder à du contenu illégal. Le deuxième épisode s'amorce donc par l'exploration des outils qui permettent de trouver de la pornographie juvénile. Quand les outils traditionnels ne suffisent plus à obtenir des contenus satisfaisants, le consommateur explorera les lieux virtuels et entrera dans une étape de socialisation. Le troisième épisode se caractérise par une immersion amenant l'utilisateur à avoir des interactions plus systématiques avec d'autres internautes afin d'accéder à des contenus plus rares, et considérés par conséquent comme plus intéressants. En plus d'y trouver un intérêt sexuel, ces images, qui ont une valeur d'échange élevée, seront utilisées pour en obtenir de nouvelles. Les contacts avec les autres consommateurs peuvent se produire lors de séances de clavardage, ou simplement en prenant connaissance des conversations archivées sur Internet. C'est dans ce contexte que le consommateur apprendra comment s'alimenter en images et éviter d'être repéré par les autorités policières. L'ultime épisode est celui du passage à l'acte où l'objet de la collection devient une cible dans le monde incarné. Pour des utilisateurs, les images seront utilisées comme un instrument menant à l'agression. La pornographie servira donc à désinhiber, à séduire et à faire chanter une victime. Pour d'autres, la pornographie juvénile serait un instrument d'excitation avant la commission de l'infraction. Il peut aussi s'agir d'individus qui veulent continuer l'exploration dans les salons de clavardage pour recruter des victimes en participant à des séances de webcam et d'échanges de photos et, dans certains cas, à des rencontres dans le réel. Finalement, la structure 
du marché de l'offre et de la demande ferait en sorte que certaines personnes filmeraient leurs agressions afin de partager ces images. La reconnaissance et le statut obtenu auprès de la communauté motiveraient ces individus à passer à l'acte.

Lanalyse des scripts semble un outil intéressant pour examiner les comportements liés aux activités de pornographie juvénile. Toutefois, certaines limites méritent d'être mentionnées. D’abord, les différentes études utilisées pour recréer les scripts n'ont pas toutes été réalisées dans le même contexte. Certaines ont été faites en laboratoire, d'autres en observant des journaux d'utilisation d'Internet, alors que certaines sont basées sur des données policières. Les études ont des sources différentes, mais aussi des méthodologies variées. Ensuite, le cadre proposé dans l'analyse des comportements de consommation de pornographie juvénile suppose l'existence de seuils de satiété à chacun des épisodes. Comme s'il n'était pas suffisant de posséder du contenu, il suppose que le délinquant doive passer à l'étape de la distribution puis à l'étape de la production une fois ces seuils atteints. Or, il est possible que nombre de ces individus possèdent des caractéristiques psychologiques et sociales qui servent de barrière et réussissent à endiguer durablement l'escalade du crime. Babchishin et ses collaborateurs $(2011,2015)$ ont trouvé qu'en comparaison des agresseurs sexuels, les consommateurs de pornographie juvénile possédaient des caractéristiques plus favorables à un contrôle des pulsions (p. ex., ils sont plus éduqués, obtiennent des scores plus élevés aux tests cognitifs, consomment moins de substances illicites et ont moins souvent d'antécédents d'agressions). On note également que les consommateurs de pornographie juvénile sont nettement moins antisociaux que les agresseurs d'enfants. Dans la perspective d'un éventuel passage à l'acte sous forme d'agression sexuelle, Seto (2009) relève que les tendances antisociales représenteraient la motivation du délinquant à agir pour assouvir ses préférences sexuelles déviantes. Ainsi, l'absence de ce genre de caractéristiques pourrait aider le consommateur de pornographie juvénile à ne pas franchir une étape ultérieure. De plus, notons que tous les utilisateurs ne traversent pas la séquence des épisodes de manière linéaire pour en arriver à la dernière. Il est d'ailleurs possible qu'un certain nombre de délinquants exercent leurs activités délictuelles de manière parallèle: d'un côté, ils consomment le contenu pédopornographique et, de l'autre, si l'occasion se présente, ils agressent sexuellement des enfants. Dans ce contexte, la sollicitation sexuelle par Internet paraît une étape facultative dans la 
transition de la consommation de pornographie à l'agression sexuelle. Enfin, bien que tous les cheminements possibles aient été cernés dans cette étude, plusieurs questions demeurent sans réponse. Dans quelle proportion les individus vont-ils passer d'un script à l'autre? Quel est le taux d'abandon d'un point de basculement à l'autre? Quels sont les facteurs et les incitatifs qui favorisent l'abandon ou du moins la stabilisation des comportements au seuil d'un nouveau script? Ces questions demeurent des pistes de recherche intéressantes à approfondir au cours des prochaines années.

\section{Références}

Akers, R. L. (2011). Social learning and social structure: A general theory of crime and deviance. New Brunswick, NJ: Transaction Publishers.

Aslan, D. (2011). Critically evaluating typologies of Internet sex offenders: A psychological perspective. Journal of Forensic Psychology Practice, 11(5), 406431.

Babchishin, K. M., Hanson, K. R. et Hermann, C. A. (2011). The characteristics of online sex offenders: A meta-analysis. Sexual Abuse: A Journal of Research and Treatment, 23(1), 92-123. http://doi.org/10.1177/1079063210370708

Babchishin, K. M., Hanson, R. K. et VanZuylen, H. (2015). Online child pornography offenders are different: A meta-analysis of the characteristics of online and offline sex offenders against children. Archives of Sexual Behavior, 44, 45-66. doi : 10.1007/s10508-014-0270-x.

Beauregard, E., Proulx, J., Rossmo, K., Leclerc, B. et Allaire, J. F. (2007). Script analysis of the hunting process of serial sex offenders. Criminal Justice and Behavior, 34(8), 1069-1084. http://doi.org/10.1177/0093854807300851

Belk, R. W. (1994). Collectors and collecting. Dans S. M. Pearce, Interpreting Objects and Collections (p. 317-326). Londres, Royaume-Uni/New York, NJ: Routledge.

Belk, R. W., Wallendorf, M., Sherry, J. F. et Holbrook, M. B. (1991). Collecting in a consumer culture. Dans R. W. Belk (dir.), SV-Highways and Buyways: Naturalistic Research from the Consumer Behavior Odyssey (p. 1-29). Provo, UT: Association for Consumer Research.

Bennett, R. et Gates, D. (1991). Relationship between pornography and extrafamilial child sex abuse. Police Chief, 14-20.

Briggs, P., Simon, W. T. et Simonsen, S. (2011). An exploratory study of Internetinitiated sexual offenses and the chat room sex offender: Has the Internet enabled a new typology of sex offender? Sexual Abuse: A Journal of Research and Treatment, 23(1), 72-91. http://doi.org/10.1177/1079063210384275

Carr, A. (2004). Internet traders of child pornography and other censorship offenders in New Zealand. Wellington, Nouvelle-Zélande: Department of Internal Affairs Te Tari Taiwhenua. 
Carr, J. (2001). Child Pornography: Theme paper for the 2nd World Congress on Commercial Sexual Exploitation of Children. Londres, Royaume-Uni: Action for Children.

Castells, M. (2002). The Internet galaxy: Reflections on the Internet, business, and society. Oxford, Royaume-Uni: Oxford University Press on Demand.

Clarke, R. V. et Newman, G. (2006). Outsmarting the Terrorists. Westport, CT: Praeger Publishers.

Cooper, A., Delmonico, D. L. et Burg, R. (2000). Cybersex users, abusers, and compulsives: New findings and implications. Sexual Addiction et Compulsivity, 7(1-2), 5-29. http://doi.org/10.1080/10720160008400205

Copes, H., Hochstetler, A. et Cherbonneau, M. (2012). Getting the upper hand: Scripts for managing victim resistance in carjackings. Journal of Resedrch in Crime and Delinquency, 49(2), 249-268. http://doi.org/10.1177/0022427810397949

Cornish, D. (1994). The procedural analysis of offending and its relevance for situational prevention. Crime Prevention Studies, 3, 151-196.

Corriveau, P. (2010). Les groupes de nouvelles à caractère pédopornographique: Une sous-culture de la déviance. Déviance et Société, 34(3), 381-400. http://doi.org/10.3917/ds.343.0381

Corriveau, P. et Fortin, F. (2011). Cyberpédophiles: et autres agresseurs virtuels. Montréal, Québec: VLB.

DeHart, D., Dwyer, G., Seto, M. C., Moran, R., Letourneau, E. et SchwarzWatts, D. (2016). Internet sexual solicitation of children: A proposed typology of offenders based on their chats, e-mails, and social network posts. Journal of Sexual Aggression. http://dx.doi.org/10.1080/13552600.2 016.1241309

Deslauriers-Varin, N. et Beauregard, E. (2010). Victims' routine activities and sex offenders' target selection scripts: A latent class analysis. Sexual abuse: A Journal of Research and Treatment, 22(3), 315-342.

End child prostitution, child pornography and trafficking of children for sexual purposes (ECPAT) (2002). Child Pornograpby: Frequently asked questions about

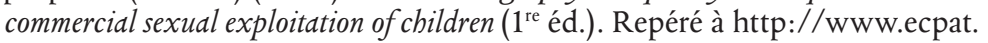
net/eng/csec/faq.

End child prostitution, child pornography and trafficking of children for sexual purposes (ECPAT) (2007, Septembre). Questions et Réponses. Repéré à http:// www.ecpat.lu/questions_fr.pdf

Fillieule, R. et Montiel, C. (1997). La pédophilie. Paris, France: Institut des Hautes études de la sécurité intérieure.

Finkelhor, D. (1984). Child sexual abuse: New research and theory. New York, NY: Free Press.

Forde, P. et Patterson, A. (1998). Paedophile Internet activity. Trends et Issues in Crime and Criminal Justice, (Novembre 1998), 6.

Formanek, R. (1991). Why they collect: Collectors reveal their motivations. Journal of Social Behavior and Personality, 6, 1-12.

Fortin, F. (2013). Usenet newsgroups, child pornography and the role of participants. Dans C. Morselli (dir.), Illicit networks (p. 231-246). Oxford, Royaume-Uni: Routledge. 
Fortin, F. (2014). C'est ma collection mais c'est bien plus que ça: Analyse des processus de collecte et de l'évolution des images dans les collections de pornographie juvénile (Thèse de doctorat inédite). Université de Montréal.

Fortin, F. et Lanthier, V. (2013). Leurre informatique: auteurs, victimes et environnement technologique. Dans F. Fortin (dir.), Cybercriminalité: Entre inconduite et crime organisé (p. 135-155). Montréal, Québec: Presses Internationales Polytechnique.

Fortin, F. et Lapointe, S. (2002, Juin). Internet qu'est-ce que ça change? Usages problématiques et criminels d'Internet. Conférence donnée au congrès de l'Association des médecins psychiatres, Mont-Tremblant, Québec, Canada.

Fortin, F. et Roy, J. (2006). Profils des consommateurs de pornographie juvénile arrêtés au Québec: L'explorateur, le pervers et le polymorphe. Criminologie, $39(1), 1-22$.

Ghernaouti-Hélie, S. (2007). Cybercriminalité et sécurité intérieure: État des lieux et éléments de prévention. Dans M. Cusson, B. Dupont et F. Lemieux (dir.). Traité de sécurité intérieure (p. 246-259). Montréal, Québec: Hurtubise $\mathrm{HMH}$.

Griffin-Shelley, E. (2014). Sex and Love Addicts, Who Sexually Offend: Two Cases of Online Use of Child Pornography (Child Sexual Abuse Images). Sexual Addiction or Compulsivity, 21(4), 322-341.

Holt, T. J. Blevins, K. R. et Burkert, N. (2010). Considering the Pedophile Subculture Online. Sexual Abuse: a Journal of Research and Treatment, 22(1), 3-24. http://doi.org/10.1177/1079063209344979

Houtepen, J. A. B. M., Sijtsema, J. J. et Bogaerts, S. (2014). From child pornography offending to child sexual abuse: A review of child pornography offender characteristics and risks for cross-over. Aggression and Violent Behavior, 19, 466-473. doi : 10.1016/j.avb.2014.07.011

Howitt, D. (1995). Paedophiles and sexual offences against children. Chischester, Royaume-Uni: Wiley Chichester.

Jensen, R. E. (2010). A content analysis of youth sexualized language and imagery in adult film packaging, 1995-2007. Journal of Children and Media, 4(4), 371-386. doi : 10.1080/17482798.2010.510005

Klain, E. J., Davies, J. H. J. et Hicks, M. M. A. (2001). Child pornography: The Criminal-Justice-System response. The National Center for Missing and Exploited Children (NCMEC), 1-168.

Knight, S. A. et Spink, A. (2008). Toward a web search information behavior model. Dans A. Spink et M. Zimmer (dir.), Web Search (p. 209-233). Berlin, Allemagne: Spring-Verlag.

Krone, T. (2004). A typology of online child pornography offending. Trends $\sim$ Issues in Crime and Criminal Justice, 279, 1-6.

Lanning, K. V. (2010). Child molesters: A behavioral analysis. NCMEC. Repéré à http://www.missingkids.com/en_US/publications/NC70.pdf

Lanning, K. V. et Burgess, A. W. (1984). Child pornography and sex rings. Washington DC: Federal Bureau of Investigation, US Department of Justice.

Leclerc, B., Wortley, R. et Smallbone, S. (2011). Getting into the script of adult child sex offenders and mapping out situational prevention measures. Jour- 
nal of Research in Crime and Delinquency, 48(2), 209-237. http://doi.org/ 10.1177/0022427810391540

Lee, A. F., Li, N. C., Lamade, R., Schuler, A. et Prentky, R. A. (2012). Predicting hands-on child sexual offenses among possessors of Internet child pornography. American Psychological Association, 18(4), 644-672.

Long, M. L., Alison, L. A. et McManus, M. A. (2013). Child pornography and likelihood of contact abuse: A comparison between contact child sexual offenders and noncontact offenders. Sexual Abuse: A Journal of Research and Treatment, 25(4), 370-395.

Marshall, W. L. (1988). The use of sexually explicit stimuli by rapists, child molesters, and nonoffenders. Journal of Sex Research, 25(2), 267-288.

McLaughlin, J. F. (2000). Cyber child sex offender typology. Knight Stick: Publication of the New Hampshire Police Association, 51, 39-42.

Middleton, D., Mandeville-Norden, R. et Hayes, E. (2009). Does treatment work with internet sex offenders? Emerging findings from the Internet Sex Offender Treatment Programme (i-SOTP). Journal of Sexual Aggression, 15(1), 5-19. http://doi.org/10.1080/13552600802673444

Mitchell, K. J., Wolak, J. et Finkelhor, D. (2005). Police posing as juveniles online to catch sex offenders: Is it working? Sexual Abuse: A Journal of Research and Treatment, 17(3), 241-267. http://doi.org/10.1177/107906320501700302

Morselli, C. et Roy, J. (2008). Brokerage qualifications in ringing operations. Criminology, 46(1), 71-98. http://doi.org/10.1111/j.1745-9125.2008.00103.x

Muensterberger, W. (1994). Collecting: An unruly passion. Princeton, NJ: Princeton University Press.

Murray, J. L. et Skoog, D. M. (1998). Innocence exploited: Child pornography in the electronic age. Ottawa, Ontario: Canadian Police College et Université de Winnipeg.

O'Brien, S. (1983). Child pornography. Dubuque, IA: Kendall Hunt Pub Co.

Ouellet, I. (2008, Novembre). Exploitation sexuelle des enfants sur Internet. Présenté à L'intersectoriel, pour des actions réalistes et efficaces auprès des victimes d'agression sexuelle. Québec, Canada.

Ouimet, M. (2006). Réflexions sur Internet et les tendances de la criminalité. Criminologie, 39(1), 7. http://doi.org/10.7202/013123ar

Philaretou, A. G., Mahfouz, A. Y. et Allen, K. R. (2005). Use of Internet pornography and men's well-being. International Journal of Men's Health, 4(2), 149-169.

Prichard, J., Watters, P. A. et Spiranovic, C. (2011). Internet subcultures and pathways to the use of child pornography. Computer Law $\mathcal{F}$ Security Review, 27(6), 585-600. http://doi.org/10.1016/j.clsr.2011.09.009

Proulx, J. et Ouimet, M. (1995) Criminologie de l'acte et pédophilie. Revue internationale de criminologie et de police technique, 48, 294-310.

Proulx, J., Perreault, C. et Ouimet, M. (1999). Pathways in the offending process of extrafamilial sexual child molesters. Sexual Abuse: A Journal of Research and Treatment, 11(2), 117-129.

Quayle, E. et Taylor, M. (2001). Child seduction and self-representation on the Internet. CyberPsychology \& Behavior, 4(5), 597-608. 
Quayle, E. et Taylor, M. (2002). Child pornography and the Internet: Perpetuating a cycle of abuse. Deviant Behavior, 23(4), 331-361. http://doi. org/10.1080/01639620290086413

Rettinger, L. J. (2000). La relation entre la pornographie juvénile et les infractions sexuelles contre les enfants: Une analyse documentaire. Ottawa, Ontario: Ministère de la Justice du Canada.

Roy, J. (2004). Étude exploratoire des évènements et des caractéristiques des individus mis en cause dans des cas de possession et de distribution de matériel pornographique juvénile sur Internet (Mémoire de maîtrise inédit). Université de Montréal.

Schank, R. C. et Robert, P. A. (1975). Scripts, plans, and knowledge. New Haven, CT: Yale University.

Sellier, H. (2003). Innocence-en-danger.com: Internet: le paradis des pédophiles. Paris, France: Pion.

Seto, M.C. (2009, Avril). Assessing the risk posed by child pornography offenders. Papier présenté pour le G8 Global Symposium, University of North Carolina, Chapel Hill.

Seto, M. C., Hanson, R. K. et Babchishin, K. M. (2011). Contact sexual offending by men with online sexual offences. Sexual Abuse: A Journal of Research and Treatment, 23, 124-145. doi : 10.1177/1079063210369013

Steel, C. M. S. (2009). Web-Based Child Pornography. International Journal of Digital Crime and Forensics, 1(4), 58-69. http://doi.org/10.4018/ jdcf.2009062405

Strano, M., Germani, P., Gotti, V. et Errico, G. (2003). La diagnosi clinica e la terapia della pedofilia. Telematic Journal of Clinical Criminology. Repéré à http://www.pornodipende.it/PEDOFILIA/Pedofilia \% 20diagnosi \% 20e \% 20terapia.pdf

Sutherland, E. H. (1947). Principles of Criminology: $4^{e} e ́ d$. Philadelphie, PN: Lippincott.

Tate, T. (1990). Child pornography: An investigation. Londres, Royaume-Uni: Methuen London.

Taylor (2001, Octobre). La pédopornographie, Internet et les infractions. Ministère de la Justice - Division de la recherche et de la statistique du ministère de la Justice du Canada (2001) - congrès transfrontalier - la frontière CanadaÉtats-Unis: Une réalité changeante séance sur Internet et la pédopornographie. Rapport de congrès. Vancouver, Canada.

Taylor, M., Quayle, E. et Holland, G. (2001). La pornographie infantile, l'Internet et les comportements délinquants. ISUMA The Canadian Journal of Policy Research, 2(2), 1-12.

Taylor, M. et Quayle, E. (2003). Child pornography: An Internet crime. New York, NY: Brunner-Routledge.

Temporini, H. (2012). Child pornography and the Internet. Psychiatric Clinics of NA, 35(4), 821-835. http://doi.org/10.1016/j.psc.2012.08.004

Twohig, M. P., Crosby, J. M. et Cox, J. M. (2009). Viewing Internet pornography: For whom is it problematic, how, and why? Sexual Addiction of Compulsivity, 16(4), 253-266. http://doi.org/10.1080/10720160903300788 
Warren, J. I., Dietz, P. E. et Hazelwood, R. R. (2013). Aggression and violent behavior. Aggression and Violent Behavior, 18(6), 666-672. http://doi.org/ 10.1016/j.avb.2013.07.020

Wolak, J., Mitchell, K. J. et Finkelhor, D. (2003). Escaping or connecting? Characteristics of youth who form close online relationships. Journal of Adolescence, 26(1), 105-119.

Wortley, R. K. et Smallbone, S. (2006). Child pornography on the internet. Problem-Oriented Guides for Police Problem-Specific Guides Series, (41).

\title{
From legal pornography to sexual assault: sexual cyber-offender activity scripts
}

\begin{abstract}
Scripts theory makes it possible to understand the particular sequence in which different types of crime may be committed. While researchers have traditionally been interested in crimes against property and individuals, this study analyzes a new form of crime, that committed on the Internet. Based on a review of the scientific literature, this article examines how viewing and creating child pornography can be understood dynamically and suggests that a motivated child pornography offender, in acquiring new knowledge and techniques, goes through many stages that ultimately lead to sexual abuse of children. It is important to note that only a small proportion of individuals who follow the steps described in the script will move on to the next step: the focus here is on the context and not on any causality between the steps, as the latter has not been demonstrated. We look at the offenders' path from consumption of adult pornography, to consumption of child pornography, to engaging in the distribution of child pornography, to child luring, and, finally, to engaging in child sexual abuse and production of child pornography. The limits and implications of the study are discussed.
\end{abstract}

KEYWORDS - Online sexual offenders, scripts, child pornography, child molesters.

\section{De la pornografía legal a la agresión sexual. Los scripts de las actividades de los ciberdelincuentes sexuales}

RESUMEN - La teoría de los scripts permite una comprensión secuencial de la criminalidad vista desde diferentes formas. Mientras que tradicionalmente los investigadores se interesan en los delitos contra los bienes y contra la persona, el presente estudio apunta a un análisis de una nueva forma de criminalidad, es decir, aquélla cometida a través de Internet. El objetivo del presente artículo es examinar, a partir de una recensión de escritos científicos, la manera en la cual el consumo de pornografía juvenil podría ser entendido según una perspectiva dinámica. Se sugiere que el consumidor de pornografía juvenil motivado, adquiere nuevos conocimientos técnicos y atraviesa varias etapas y obstáculos que lo conducen por último a la agresión sexual de niños. Sin embargo, es importante resaltar que sólo una pequeña parte de los individuos entraran en las etapas subsecuentes del scripts, poniendo el acento en el contexto, y no en los lazos de causalidad que unen las etapas, ya que su existencia debe ser demostrada. Detallaremos especificamente los recorridos del consumo de 
pornografía adulta hacia la pornografía juvenil, la distribución de este tipo de material, el engaño de menores y, finalmente, la concretización, o sea el abuso de niños y la producción de pornografía juvenil. Los límites y las implicaciones del estudio serán establecidos.

PALABRAS CLAVE - Ciberdelincuentes sexuales, scripts, pornografía juvenil, agresores sexuales de niños. 